\title{
The Effect of Contour Closure on the Rapid Discrimination of Two-Dimensional Shapes
}

\author{
JAMES ELDER,* STEVEN ZUCKER* \\ Received 24 February 1992; in revised form 2 September 1992
}

\begin{abstract}
An outline drawing often serves as an excellent depiction of a visual scene. Somehow, our visual system can form two- and three-dimensional percepts solely from one-dimensional contour information. In mathematies, contour closure plays a key role in bridging this dimensional gap, however in perception the link between closure and shape is unclear. To better understand this relationship, we devised a set of visual search experiments in which subjects discriminate outline figures by means of their two-dimensional shape. By modulating the degree of closure of the outlines, we show that twodimensional shape processing is rapid for closed stimuli but slow for open stimuli. We further show that search can be characterized as a smooth, monotonic function of the degree of closure, supporting the notion of a perceptual closure continuum.
\end{abstract}

Contour Shape Topology Perceptual organization Visual search

\section{INTRODUCTION}

Amongst the many contours in a natural image, there will be some which project from the boundaries of objects. Integrating information from these occlusion contours allows the inference of two- and three-dimensional shape properties. The trick is to pick the right contours to integrate, so that non-occlusion contours, or occlusion contours from distinct boundaries, are not integrated into representations of non-existent objects.

The hypothesis motivating this work is that this process of selective integration is based upon contour closure. This perceptual closure is presumed to have some correspondence to mathematical and intuitive notions of closure, but also to have properties specific to a perceptual context.

The importance of contour closure to perception was observed and discussed by Gestalt psychologists in the 1920s and 1930s (Fig. 1):

"Ordinary lines, whether straight or curved, appear as lines and not as areas. They have shape, but they lack the difference between an inside and an outside... . If a line forms a closed, or almost closed, figure, we see no longer merely a line on a homogeneous background, but a surface figure bounded by the line" (Koffka, 1935).

The goal of this paper is to make explicit the connection between contour closure and the inference of multidimensional shape properties. We begin with the observation that the boundary of an unoccluded object with a simply connected surface projects as a simple,

*McGill Research Centre for Intelligent Machines, McGill University, 3480 University Street, Montréal, Québec, Canada H3A 2 A7. closed contour in a retinal image. A classical result in topology known as the Jordan curve theorem states that such a contour partitions the plane into two sets: an inside (figure) and an outside (ground). This partitioning is crucial because it allows the definition of two-dimensional shape properties such as curvature sign, concavities, convexities, narrowings and bulges. Such properties have in turn been shown to impose strong constraints on the shape of the three-dimensional surfaces in the scene (Biederman, 1988; Koenderink, 1984; Lowe, 1985; Marr, 1982). Thus, computationally, notions of contour closure and shape are deeply entwined: contour closure makes the inference of shape from contour possible.

While in topology there is no concept of partial contour closure (a curve is either closed or it is not), in perception, shape can still be perceived even when the bounding contour is fragmented by occlusion, shadow or low reflectance contrast. What then is the role of closure in the perception of shape?

Our hypothesis is that in perception the notion of a closure continuum exists and that it is this perceptual closure which mediates shape perception. This hypothesis connecting closure to shape has never been tested: contemporary research has instead divorced these two notions, characterizing closure as an "emergent feature", "completely abstractable from shape" (Treisman \& Paterson, 1984).

This view has led to visual search and texture discrimination experiments designed to determine whether closed shapes are easily distinguished from open shapes (Caelli, Julesz \& Gilbert, 1978; Julesz, 1980; Treisman \& Gormican, 1988; Treisman \& Paterson, 1984). A typical visual search task is to detect a closed "target" in a field of open "distractors". These experiments are normally 


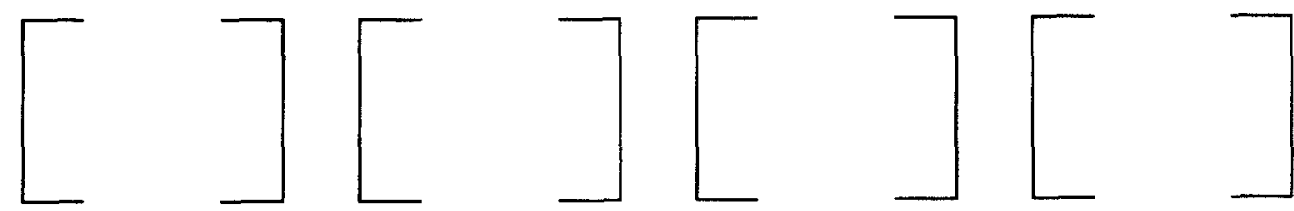

FIGURE 1. Gestalt illustration of the predominance of closure over proximity in determining the perceptual organization of a figure. After Koffka (1935)

interpreted in terms of models which distinguish spatially parallel feature detectors from spatially serial processes which compose detected features into more complicated forms (Cave \& Wolfe, 1990; Treisman \& Gelade, 1980). If the time for target detection is independent of the number of distractors in the display, it is assumed that the target and the distractor differ in the features they possess.

Interpretation of these experiments is complicated by the fact that presence or absence of closure is never the only difference between the target and distractor stimuli. Thus, while some researchers have concluded from these experiments that closure is a feature (Caelli et al., 1978; Pomerantz, Sager \& Stoever, 1977; Treisman \& Gormican, 1988; Treisman \& Paterson, 1984), others claim that line terminations are the salient features, and it is only their absence which registers closure (Julesz, 1980; Julesz \& Bergen, 1987).

Our goal was not to determine whether closure is or is not a perceptual feature, but rather to test our hypothesis that a closure continuum mediates the perception of shape. We have thus designed a set of visual search experiments in which discrimination is based not on the presence or absence of closure, but on the two-dimensional shape of the target and distractor. By modulating the degree of closure of both the target and distractor in tandem, we have been able to characterize the influence of closure on shape perception, and to isolate certain properties of this perceptual closure.

\section{METHODS}

Visual search displays were created on a $60 \mathrm{~Hz}$, noninterlaced color Amtron monitor, driven by a Symbolics 3640 computer. Subjects sat in a dimly lit room, approx. $1 \mathrm{~m}$ from the screen. A $7 \times 7^{\circ}$ square display window of luminance $11 \mathrm{~cd} / \mathrm{m}^{2}$ was positioned in the center of the screen against a background luminance of $0 \mathrm{~cd} / \mathrm{m}^{2}$. Stimuli were drawn in the display window at $72 \mathrm{~cd} / \mathrm{m}^{2}$.*

All stimuli were approx. $0.5 \times 0.5^{\circ}$ in size. Placement of the stimuli within the display window was based on a regular $5 \times 5$ grid with nodes separated by $1.4^{\circ}$ in the horizontal and vertical directions. Nodes were selected pseudorandomly, and the precise position of each stimulus was pseudorandomly selected from the set of positions within a $0.3^{\circ}$ horizontal and vertical distance from the selected node. Stimuli were thus separated by

*A different set of luminances was used for experiments exploring the dependence of closure on contrast. See Section 6 for details.

†In the last experiment, only one display size is used. See Section 7 for details. at least $0.8^{\circ}$ from center to center, and by at least $0.3^{\circ}$ from tip to tip. Each stimulus was randomized in orientation.

Displays contained either 7, 15 or 23 distractor stimuli and one target (display sizes of 8,16 or 24 stimuli). $\dagger$ The procedure is illustrated in Fig. 2. First, an example of the target for which the subject will be searching is shown [Fig. 2(a)]. The subject then presses a mouse button to trigger a sequence of 30 visual search trials (10 for each display size, randomly interleaved). In each trial, a display is presented which always contains exactly one target [Fig. 2(b)]. When the target is detected, the subject clicks a mouse button and the response time for detection is recorded. At the same time, the visual search display is replaced by a validation display in which the stimulus positions are represented by small reference dots [Fig. 2(c)]. The subject must correctly identify the target location (by clicking on the appropriate dot) for the trial to be considered valid. If an error is made, the trial is rejected, and another trial with the same display size and stimulus type is randomly inserted in the sequence to replace it.

This procedure differs from traditional methods, in which half of the displays shown to each subject contain a target and half do not (Treisman \& Gelade, 1980). In this procedure, subjects press one of two buttons, depending upon whether they perceive the target as present or absent. One advantage of our procedure is the relatively low error rates, which averaged $1.7 \%$ and were $<5 \%$ for all experiments. More importantly, we believe that the traditional method is subject to a systematic bias which our method avoids.

We have reproduced the basic results of this work using the more traditional procedure. These results, together with an analysis of the problems with traditional techniques, can be found in the Appendix.

Before each session, subjects completed a practice procedure identical to the recorded session, but including only two trials for each display condition. In addition, for every block in the recorded session, the first three trials (one for each display size) were used as practice, and the response times were not used in computing mean results, which are thus averaged over nine trials for each stimulus/display size condition.

Techniques other than visual search could be chosen to explore the role of contour closure in shape perception. For example, one could measure the time required to identify a single, briefly presented outline shape as a function of the closure of the outline.

An advantage of the visual search procedure is that it does not require recognition: the target can be located on the basis of its uniqueness alone. We feel that this may 


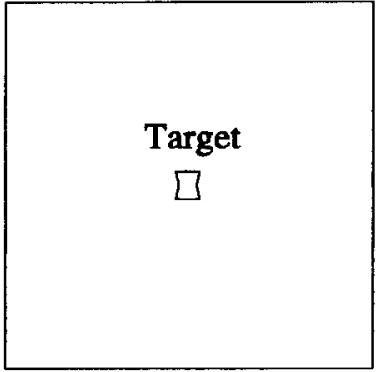

(a)

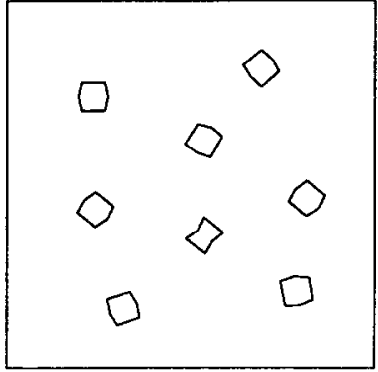

(b)

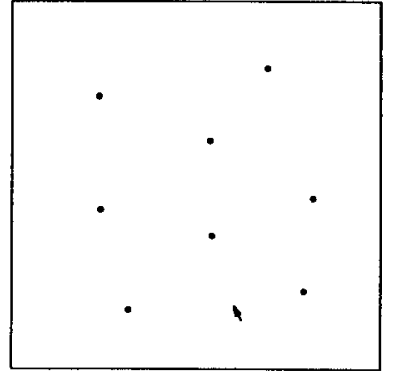

(c)

FIGURE 2. Visual search sequence.

better isolate early mechanisms for shape representation which are relatively independent of memory.

The main disadvantage of the visual search method is that it confounds the problem of forming useful shape representations with the problem of distinguishing the figures from one another. We address this issue in Section 8.

\section{SUBJECTS}

A total of 29 subjects ( 21 male, 8 female) were used for the experiments described in this paper. Between 10 and 14 subjects were used for each experiment: the exact number is stated with the results. These subjects ranged from complete naiveté to full awareness regarding the goals of the study. All subjects had normal or corrected vision. Results are averaged over all participating subjects, with error bars indicating standard error of the mean.

\section{SHAPE DISCRIMINATION FOR OPEN AND CLOSED FIGURES}

The basic stimuli [Fig. 3(a)] are composed of two unconnected but nearby contours. The contour segments are the same for both the target and distractor, which thus differ only in how the segments are placed relative to each other. In the target stimulus, they are arranged to bend inward, forming a "spindle" shape, while in the distractor stimulus they bend outward, forming a "barrel" shape. The stimuli have thus been chosen so that the discrimination must be based on measurements which are two-dimensional and extrinsic to the contour segments. That is, information from the two contour segments forming each stimulus must be grouped into a composite representation which can then be used to discriminate the target from the distractors.

With the addition of two identical line segments to each of these open figures, two closed figures are formed [Fig. 3(b)]. The length and relative spatial position and orientation of these segments is the same for each stimulus, thus by themselves they provide no direct means for discrimination or identification. Note that closing these figures endows them with new two-dimensional shape properties. For example, the closed spindle possesses two concavities and a narrowing which the closed barrel does not possess. These properties play a large role in computational theories of planar shape perception (Blum, 1973; Hoffman \& Richards, 1985; Kimia, Tannenbaum \& Zucker, 1990; Leyton, 1989) and

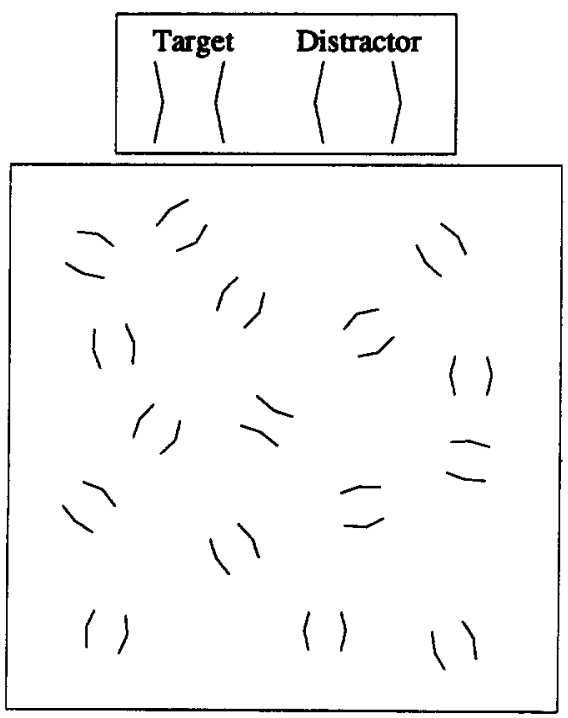

(a)

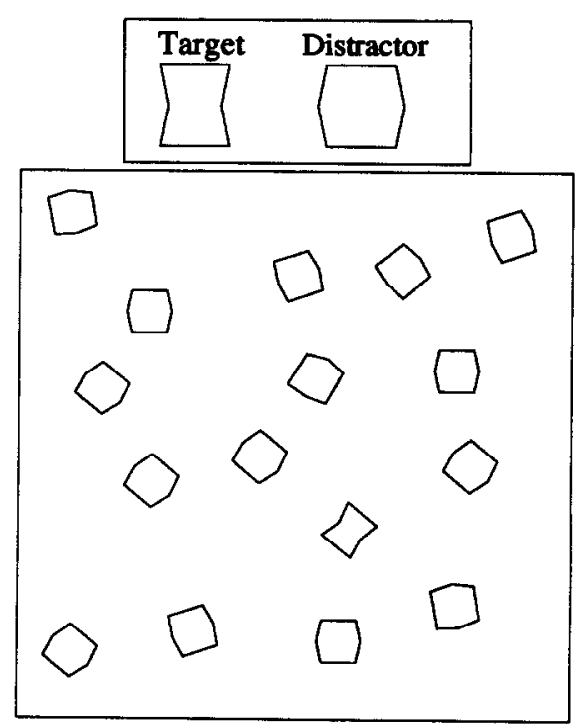

(b)

FIGURE 3. Stimuli and example displays for open and closed outline shapes. 


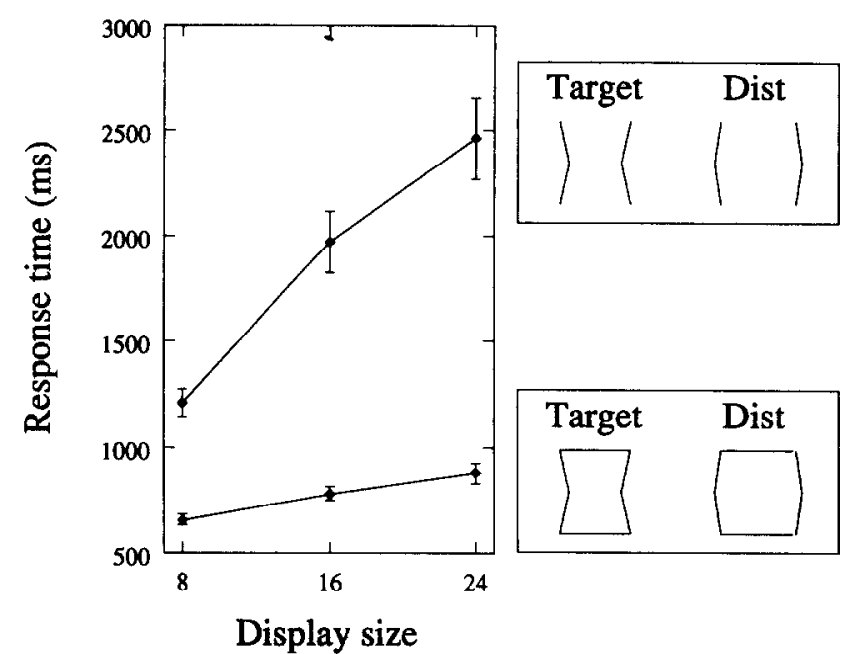

FIGURE 4. Search results for closed and open figures, averaged over 14 subjects.

so it is natural to wonder whether closure, by making these properties well-defined, will make these shapes easier to discriminate.

The results show that closing the curves dramatically enhances discriminability (Fig. 4).* While search speed for the open shapes depends strongly on the number of stimuli in the display (slope $=83 \mathrm{msec} /$ item, intercept $=555 \mathrm{msec}$ ), search for the closed shapes depends only weakly on the number of stimuli (slope $=14 \mathrm{msec} /$ item, intercept $=546 \mathrm{msec}$ ), and is within the range of what is normally considered preattentive perception (Enns \& Rensink, 1991; Julesz, 1986; Treisman \& Gormican, 1988).

In order to draw solid conclusions, however, we must examine the other changes introduced by closing the stimuli. For example, the closed contours possess four corners (orientation discontinuities), which could provide local information sufficient for discrimination. Also, the closed figures are connected. Connectedness has been proposed as an important rule of perceptual organization (Rock \& Palmer, 1990): perhaps this property of connectedness is responsible for our results.

\section{CORNERS, CONNECTEDNESS OR CLOSURE?}

Three experiments were performed which evaluate the importance of closure relative to other stimulus factors.

In the first of these (Fig. 5), the end quarters of the closing segments were removed to form stimuli possessing neither local corner information nor the property of

*Slopes and intercepts were subjected to pair-wise one-tailed $t$-tests with a chosen significance level of 0.05 . Closing the stimuli significantly reduced search slope $(P<0.005)$, but had no significant effect on the intercept estimate $(P>0.1)$.

$\uparrow$ Removing the end quarters of the closing line segments resulted in a significantly greater search slope $(P<0.025)$, but had no significant effect on the intercept estimate $(P>0.01)$.

$\ddagger$ Turning the corners outward resulted in a significantly greater search slope $(P<0.005)$ but had no significant effect on the intercept estimate $(P>0.05)$.

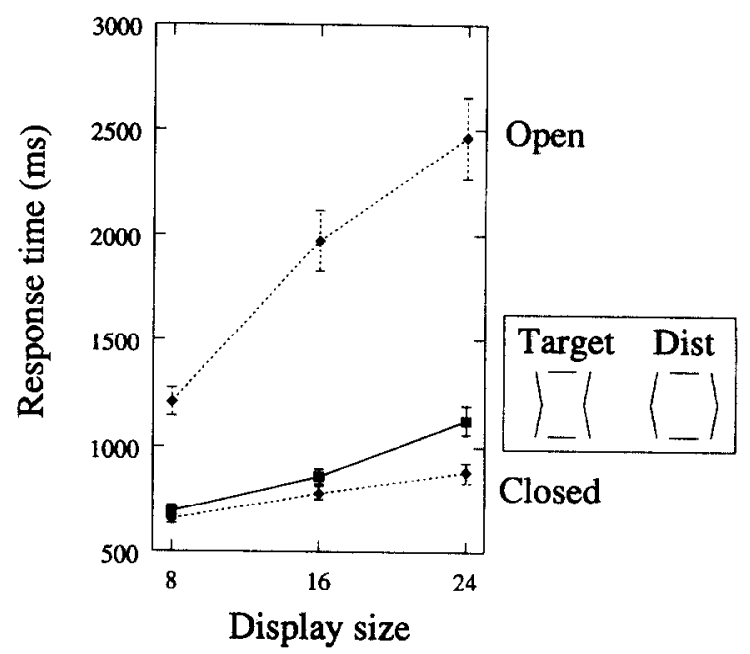

FIGURE 5. Search results for unconnected figures (14 subjects). The results for closed and open figures are shown dotted for reference.

connectedness. Although the stimulus contours are no longer topologically closed, intuitively they remain highly closed. The results confirm that while the absence of corners and connectedness does not null the effect, search performance is mildly degraded, consistent with a small decline in perceptual closure $\dagger$ (slope $=25 \mathrm{msec} /$ item, intercept $=483 \mathrm{msec}$ ).

The next experiment further investigates the role of local information in this visual search task. Two pairs of stimuli were constructed, which both possess local corner information, but differ in their degree of apparent closure (Fig. 6). The length of each horizontal segment forming a corner is one quarter of the total gap size.

The results show that, while the local information is the same in both cases, there is an immense difference in subjects' ability to discriminate the shapes. $\neq$ When the corners were oriented inward to partly close the shape, discrimination was relatively rapid (slope $=27 \mathrm{msec} /$ item, intercept $=535 \mathrm{msec}$ ). When the corners were oriented outward, discrimination was very slow (slope $=112 \mathrm{msec} /$ item, intercept $=894 \mathrm{msec}$ ). Clearly the global closure information is far more

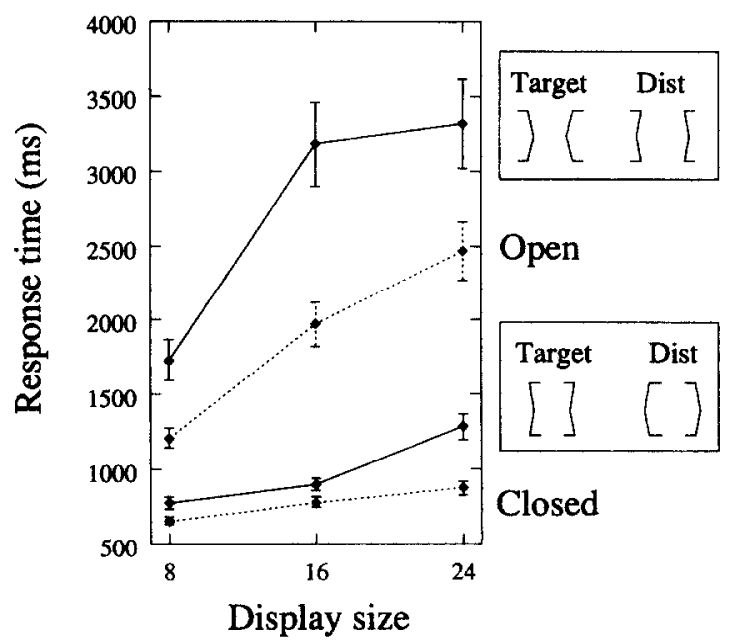

FIGURE 6. Search results for figures with inward or outward corners (14 subjects). Results for open and closed figures are shown dotted for reference 


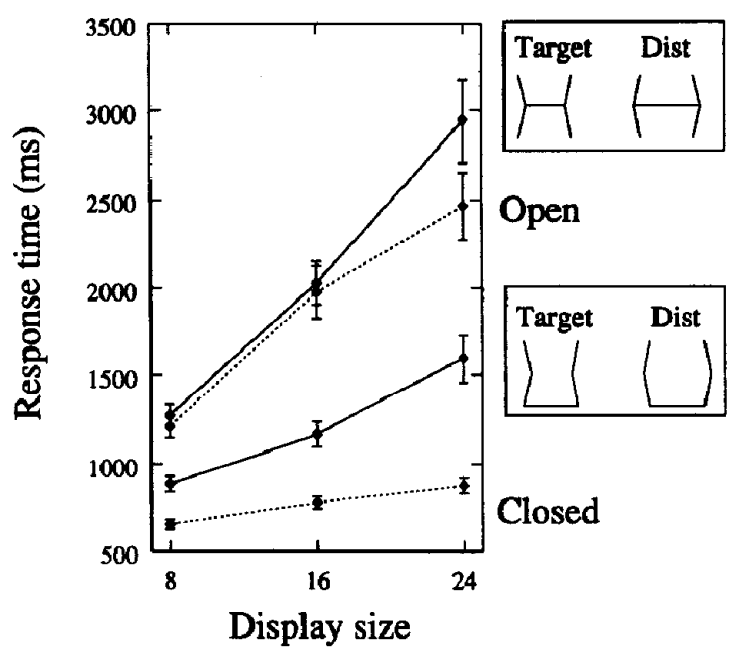

FIGURE 7. Search results for connected stimuli (14 subjects). Results for open and closed figures are shown dotted for reference.

important than these local cues in forming the representations required to discriminate the stimuli.

A third pair of experiments further investigates the difference between connectedness and closure (Fig. 7). Both experiments involve connected figures, but while the contours of one stimulus pair can be interpreted as partial object boundary projections (intermediate closure), those of the other pair are inconsistent with such an interpretation (poor closure).

Search speed for the stimuli closed at one end was intermediate between open and closed (slope $=40 \mathrm{msec} /$ item, $\quad$ intercept $=556 \mathrm{msec}$ ), while search speed for stimuli connected at the middle was slow (slope $=100 \mathrm{msec} /$ item, intercept $=466 \mathrm{msec}$ ). Connectedness was not the dominant influence here.

\section{CONTRAST REVERSAL AND CONTOUR CLOSURE}

From previous studies of contrast sign sensitivity, two classes of perceptual phenomena have emerged.

\footnotetext{
* Search slope for the stimuli connected at one end was significantly greater than that for closed stimuli $(P<0.005)$ and significantly less than that for open stimuli $(P<0.005)$. Search slope for the stimuli connected at the middle was significantly greater than that for the stimuli connected at one end $(P<0.005)$, but not significantly different from that for open stimuli $(P>0.1)$. Intercepts for the end-connected and middle-connected stimuli did not differ significantly from each other or from those for the open and closed stimuli $(P>0.1)$.

†Search using closed stimuli drawn in white yielded a $19 \mathrm{msec} /$ item slope and a 496 msec intercept. Search using closed stimuli drawn in black yielded a search slope of $14 \mathrm{msec} /$ item and an intercept of $692 \mathrm{msec}$. Search using open stimuli drawn in white yielded a $93 \mathrm{msec} / \mathrm{item}$ slope and a $340 \mathrm{msec}$ intercept. Search using open stimuli drawn in black yielded a $105 \mathrm{msec} /$ item slope and a 444 msec intercept. Stimulus contrast does not significantly affect search slope or intercept for closed or open stimuli $(P>0.1)$.

$¥$ Search slope is significantly greater than that for white stimuli $(P<0.025)$ and for black stimuli $(P<0.01)$. Intercepts do not differ significantly $(P>0.1)$.
}

Phenomena based on the short-range grouping of dots into one-dimensional structures (contours) can be destroyed by reversing the contrast of alternate dots (Glass \& Switkes, 1976; Prazdny, 1986; Zucker \& Davis, 1988; Zucker, Stevens \& Sander, 1983). A beautiful example of this, due to Glass and Switkes (1976), is shown in Fig. 8.

On the other hand, phenomena that depend upon longer-range grouping into two- or three-dimensional structures seem to be robust to contrast reversal (Prazdny, 1983; Shapley \& Gordon, 1985; Zucker, 1986). For example, Prazdny (1983) has shown that modal completion will occur for contrast-reversing contours (Fig. 9).

If closure is a bridge between one- and twodimensional structure, and between local measurements and global figural representation, into which class will it fall?

In experiments designed to answer this question, we used the four different luminance levels listed in Table 1. Level 4 , the brightest, is the luminance used to draw the figures in all of the experiments described to this point. Level 2 is the background luminance used in these experiments. Level 3 is an intermediate luminance which will be used to examine the effect of reducing figure contrast, and level 1 will be used to examine the effect of reversing contrast.

\subsection{Contrast controls}

The first experiment is a control to ensure that search speed does not depend upon whether the stimuli are drawn in white or black. The results (Fig. 10) fail to show any significant dependence of search speed upon the contrast sign of the stimuli. $\dagger$

Our next two experiments are also control experiments, designed to determine whether variation in contrast over the entire display leads to slower search speed. In both of these experiments, figures may be drawn either in black or in white (Fig. 11). In the first case, the target could be of either contrast. In the second case, the target is always white, and subjects are told this in advance. These experiments were performed only for the closed figures.

When subjects did not know the target contrast in advance (Fig. 12), search was slower than for the single contrast displays $\ddagger$ (slope $=43 \mathrm{msec} /$ item, intercept $=522 \mathrm{msec}$ ). However, when the target stimulus was always white, search was much faster (slope $=23 \mathrm{msec} /$ item, intercept $=349 \mathrm{msec}$ ) and did not

TABLE 1. Luminance values used in contrast experiments

\begin{tabular}{cc}
\hline Level & $\begin{array}{c}\text { Luminance } \\
\left(\mathrm{cd} / \mathrm{m}^{2}\right)\end{array}$ \\
\hline 1 & 0 \\
2 & 11 \\
3 & 37 \\
4 & 72 \\
\hline
\end{tabular}




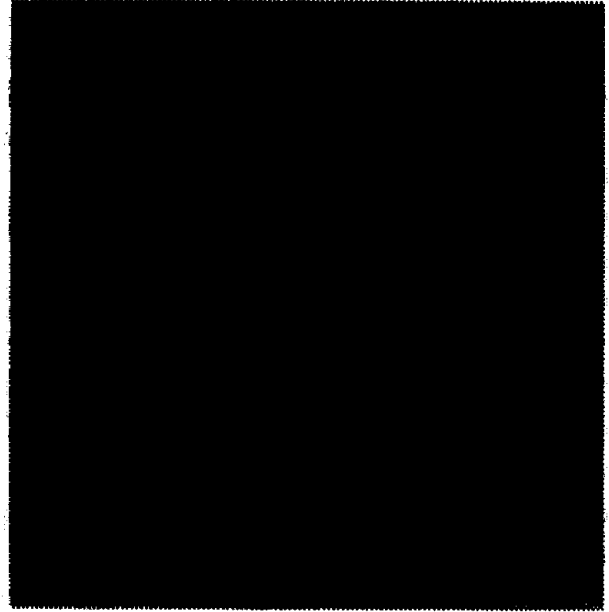

(a)

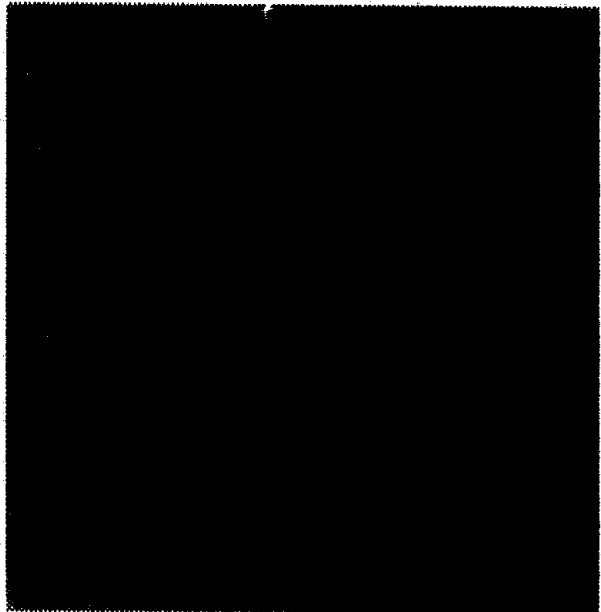

(b)

FIGURE 8. The destruction of short-range structure by contrast reversal. Pattern (a) is created by the superposition of a pattern of dots and a duplicate pattern rotated by $3 \mathrm{deg}$. Pattern (b) is identical to (a), with the duplicate pattern reversed in contrast. After Glass and Switkes (1976).

differ significantly from the single contrast search (Fig. 13).*

We conclude from these two experiments that it is primarily the uncertainty in the nature of the target that leads to slower search speed: as long as the subject knows what to look for, contrast variation across the display does not significantly affect results.

\subsection{Intra-figure contrast variation}

The control experiments have shown that search is independent of the contrast sign of the figures, and is not slowed by displays mixing figures of opposite contrast signs. We can now examine the effect of varying contrast along the contours of individual figures. The two stimulus pairs used for this experiment are shown in Fig. 14. In both pairs, the side fragments of the figures are drawn at luminance level 4. For the pair on the left, the connecting bars are drawn at luminance level 3 , resulting in a reduction of contrast, whereas for the pair on the right, they are drawn at luminance level 1 , resulting in a reversal of contrast. The results are shown in Fig. 15. While reducing the contrast results in only a mild decline in performance from the original closed figures (slope $=22 \mathrm{msec} /$ item, intercept $=450 \mathrm{msec}$ ), reversing the contrast produces results nearly identical to those for the original open figures (slope $=90 \mathrm{msec} / \mathrm{item}$, intercept $=458 \mathrm{msec})$ : contrast reversal eliminates perceptual closure. $\uparrow$ This is in conflict with recent models of shape

*Search slope for the mixed stimulus displays where the target contrast is known does not differ significantly from slopes for all-white $(P>0.1)$ or all-black $(P>0.05)$ displays. Intercept for the mixed displays does not differ from that for the all-white displays $(P>0.05)$, but is significantly less than that for the all-black displays $(P<0.005)$.

†Reducing the contrast of the closing line segments produced a significantly greater search slope $(P<0.05)$, but had no significant effect on intercept. Reversing the contrast of the closing line segments resulted in mean search slope and intercept which do not differ significantly from those for the open stimuli $(P>0.1)$.

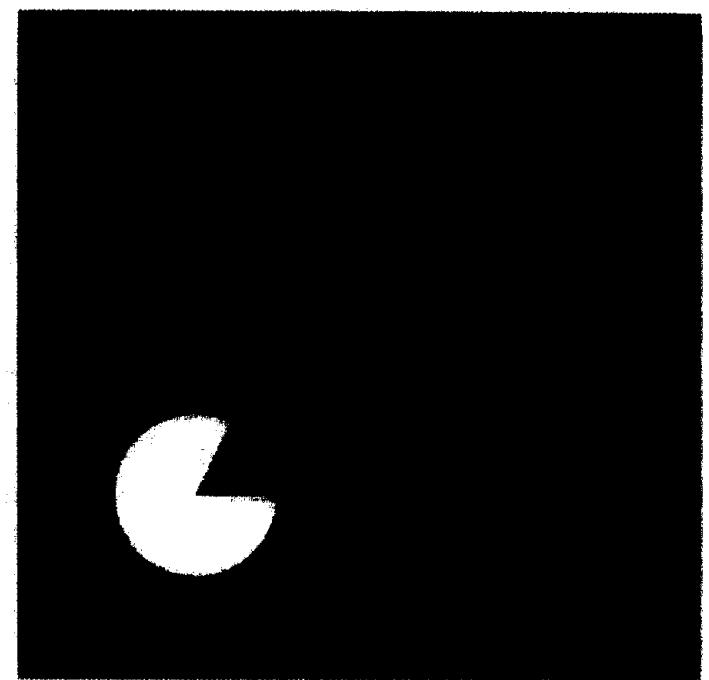

FIGURE 9. Modal completion of reversed-contrast contours.

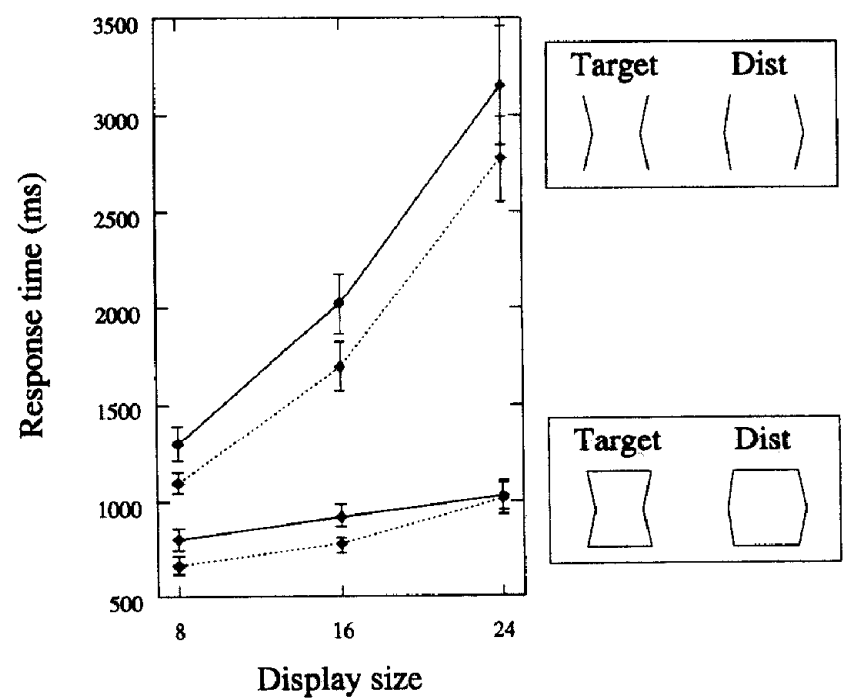

FIGURE 10. Search results for contrast control experiment (10 subjects). Results for black stimuli are shown solid, those for white stimuli are shown dotted. 


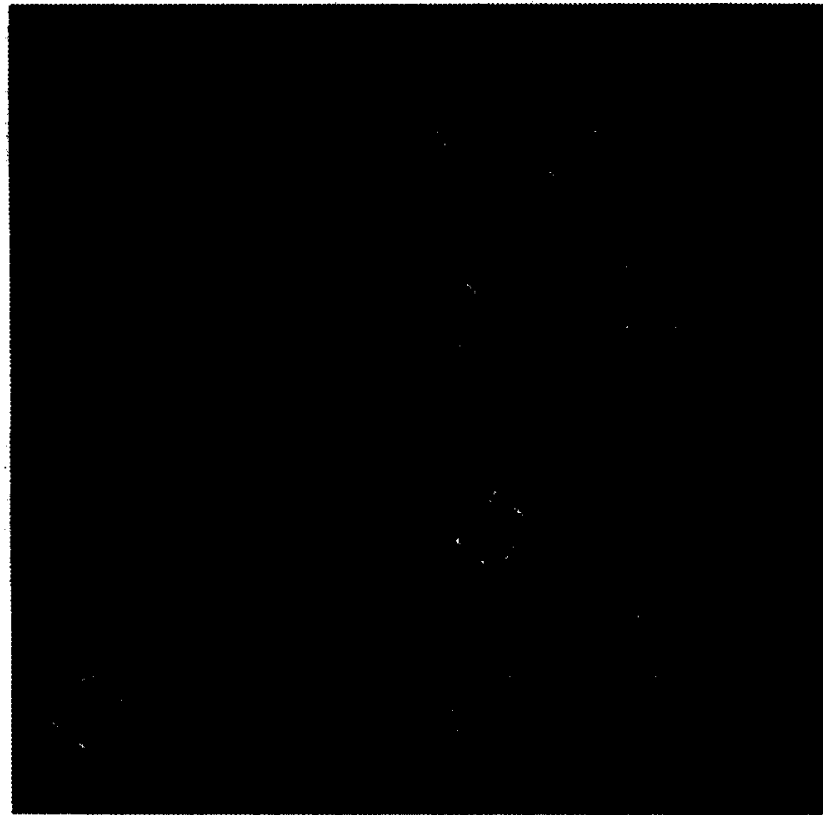

FIGURE 11. Search display with a mixture of black and white stimuli.

processing which predict that boundary grouping processes must be insensitive to contrast sign (Grossberg \& Mingolla, 1985; Shapley \& Gordon, 1985).

Subsequent experiments have shown that similar results hold when the contrast sign reverses along straight line segments of the shapes, rather than at the corners as in this experiment (Elder, 1992).

The difference in contrast sign sensitivity between these experiments and phenomena such as modal completion (Fig. 9) suggests a distinction between early shape-from-contour processes, and higher-level processes which organize the perception of multiple overlapping surfaces.

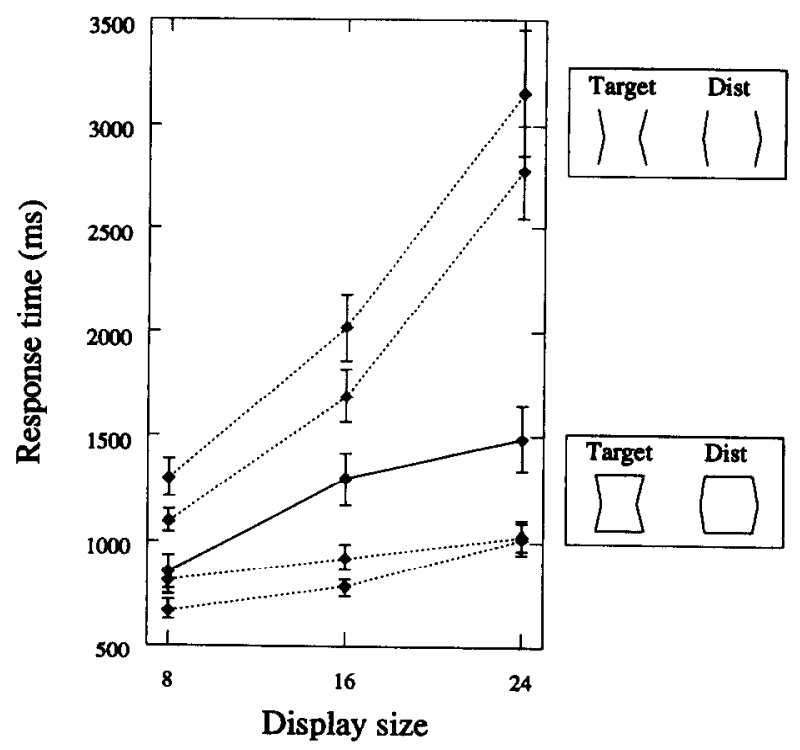

FIGURE 12. Results of search when subjects do not know the contrast sign of the target ( 10 subjects). Results for single-contrast search (either white or black) are shown dotted for reference.

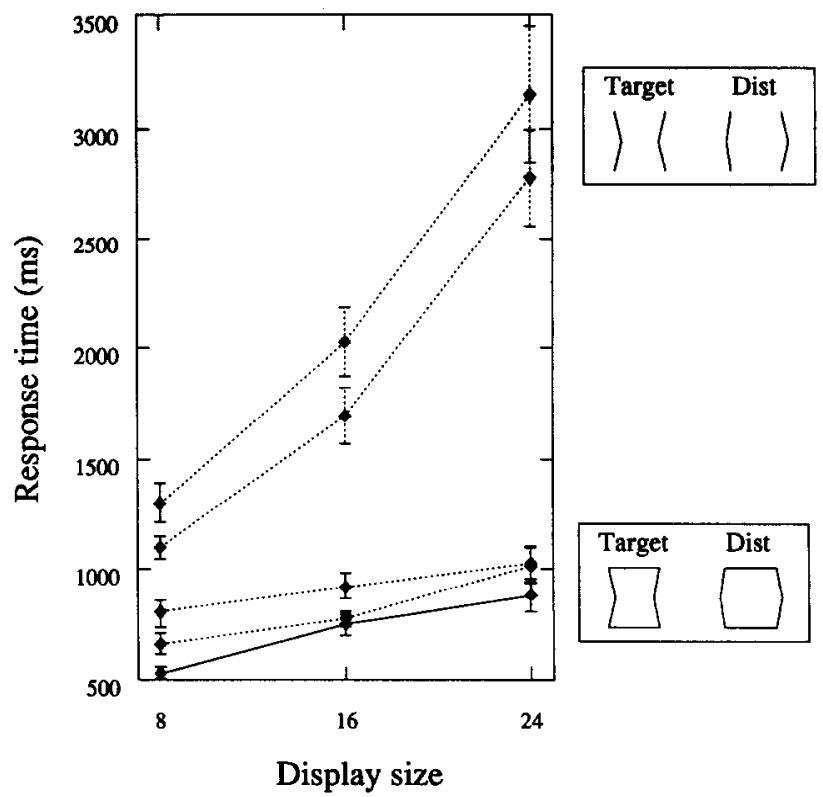

FIGURE 13. Results of search when subjects know the contrast sign of the target (10 subjects). Results for single-contrast search (either white or black) are shown dotted for reference.

\section{A CONTINUUM OF CONTOUR CLOSURE}

The experiments of Section 5 suggest that perceptual closure is not well-modeled as a topological property: when the bounding contour is fragmented, the ability of the human visual system to perceive two-dimensional shape is degraded but not destroyed.

To further clarify this, we created a new set of stimuli by incrementally adding contour to the original open barrel and spindle (Fig. 16). We conducted visual search experiments using these stimuli with a fixed display size of 16 , characterizing search speed by the mean response time for each stimulus pair. The results are shown in
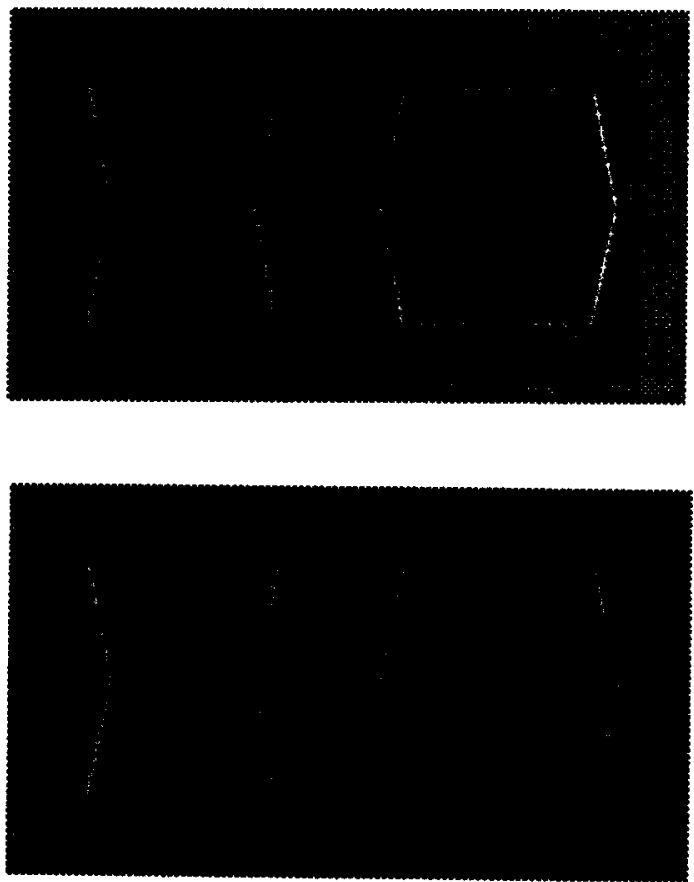

FIGURE 14. Closing the figures with reduced and reversed contrasts. 
Fig. 17, with response time plotted as a function of the number of pixels added to the open stimuli. Pixels added to form corners away from the figure are plotted in the negative abscissa range.

Intuitively, the stimuli in the high positive abscissa range have a very high degree of closure, while those in the high negative range have a very low degree of closure. The results of this experiment show that this intuition does correspond to the way closure is used to form rapid representations of shape. For stimuli that we think of as highly closed, response time is rapid, and for stimuli that we think of as poorly closed, response time is slow. The concept of a continuum of perceptual closure is highly useful here.

A linear model of response time as a function of the length of the corner extensions is inadequate, however quadratic and exponential models both provide good fits. ${ }^{*}$ Both of these models have positive curvature, indicating that contour fragment extension has a bigger impact on the absolute time required to perform the task when the degree of closure is small.

*The $\chi^{2}$ for each fit was computed using the standard deviations for each stimulus condition. Each fit involved 1080 data points (10 subjects $\times 9$ trials $\times 12$ stimulus conditions). The linear model has 2 degrees of freedom $(y=a+b x)$, the quadratic $\left(y=a+b x+c x^{2}\right)$ and exponential $\left(y=a+b \mathrm{e}^{-c x}\right)$ models have 3 . The $\chi^{2}$ for the linear model is 1120 . Since the probability $Q$ that, given a linear model, the $\chi^{2}$ would exceed 1120 is 0 , this is a poor model. The $\chi^{2}$ for the quadratic and exponential models are 1074 and 1075 respectively, both yielding $Q=1$.
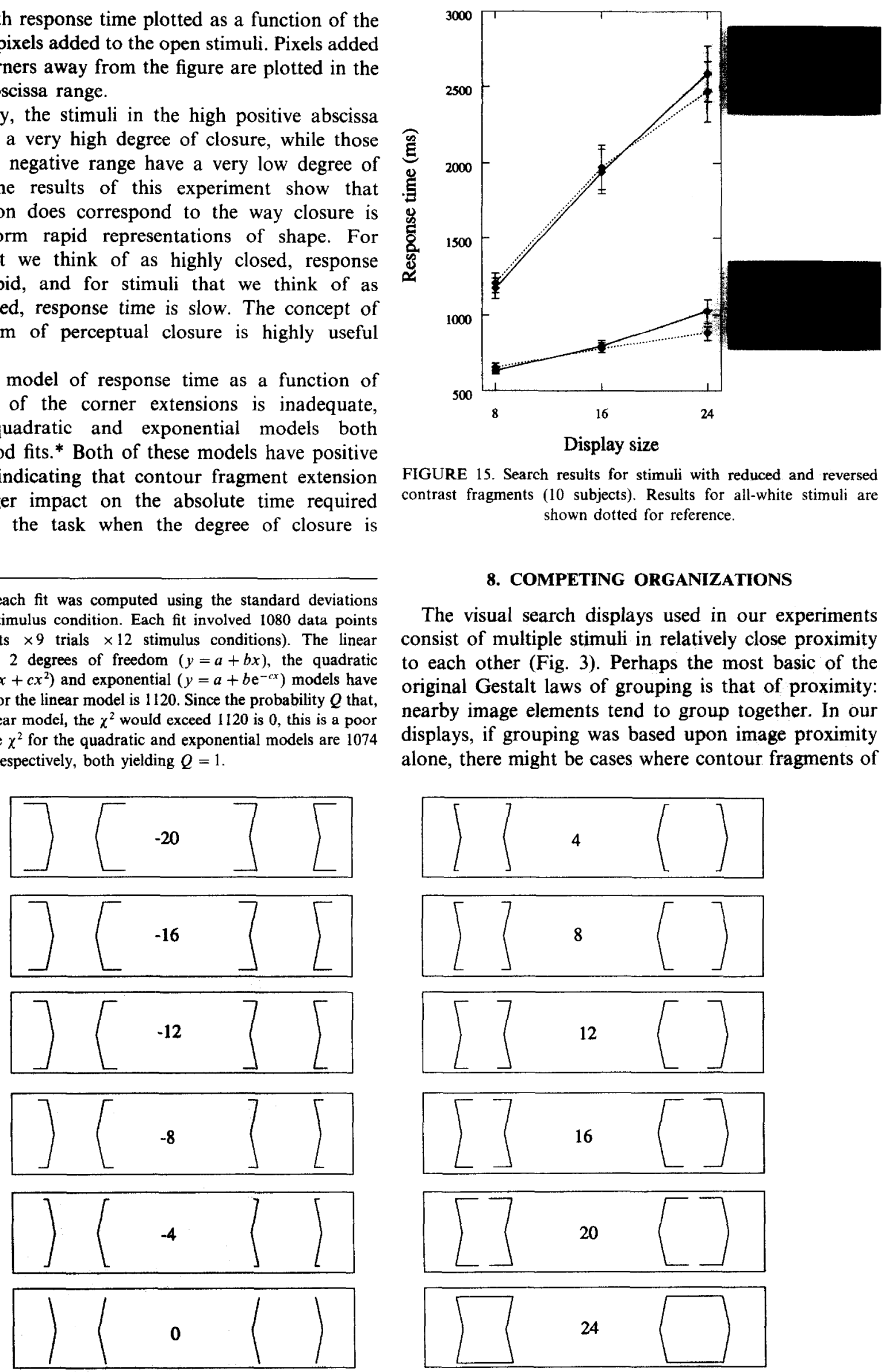

FIGURE 15. Search results for stimuli with reduced and reversed contrast fragments (10 subjects). Results for all-white stimuli are shown dotted for reference.

\section{COMPETING ORGANIZATIONS}

The visual search displays used in our experiments consist of multiple stimuli in relatively close proximity to each other (Fig. 3). Perhaps the most basic of the original Gestalt laws of grouping is that of proximity: nearby image elements tend to group together. In our displays, if grouping was based upon image proximity alone, there might be cases where contour fragments of
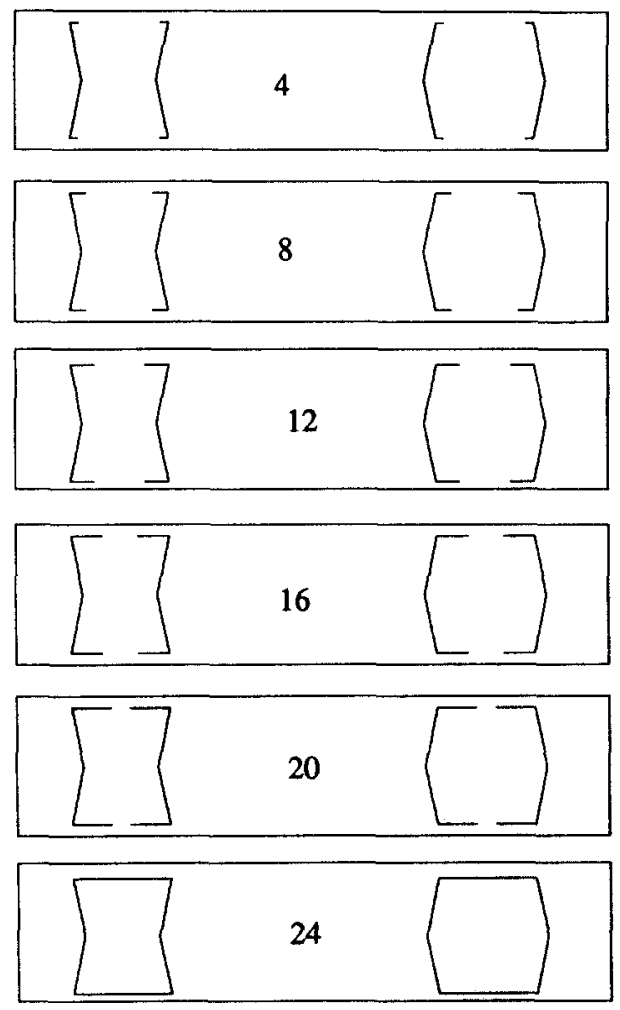

FIGURE 16. Stimuli with different degrees of closure, created from the original open stimuli. Each stimulus is mapped to an integer whose magnitude indicates the number of pixels added, and whose sign indicates whether the pixels are added to form inward or outward corners. 


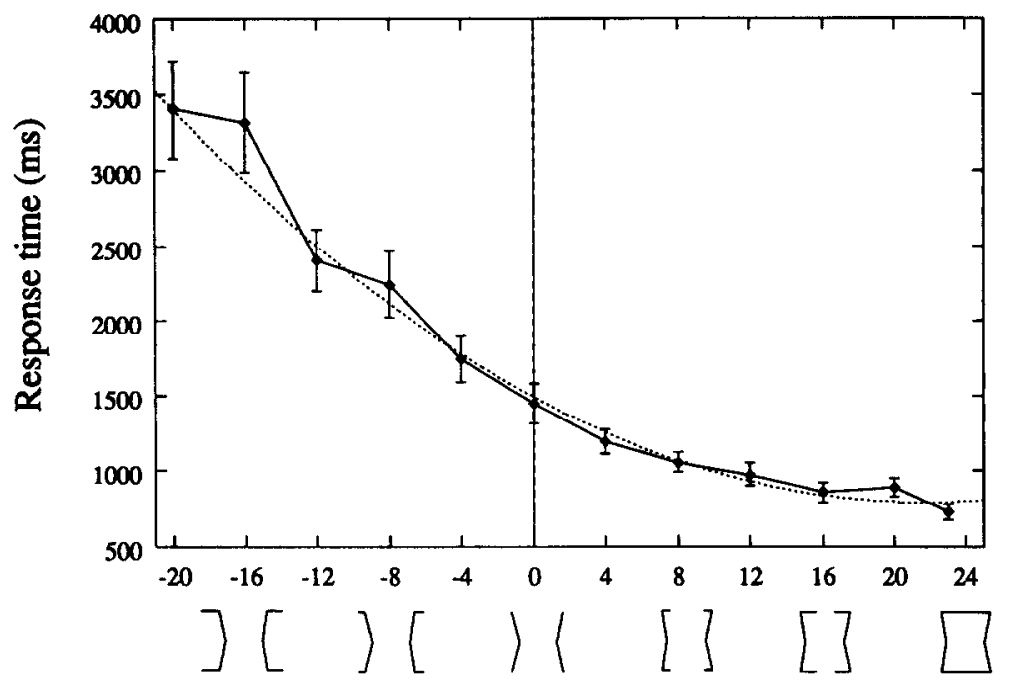

Closing pixels

FIGURE 17. Response time as a function of pixels added (10 subjects, display size $=16)$. LSE quadratic fit is shown dotted.

different stimuli would group together, forming composite representations that resemble neither the target nor the distractor. This confusion could plausibly lead to slower search, and in particular larger search slopes, for the incidence of such confusion can be expected to rise with the number and density of stimuli in the image.

It is therefore possible that the role of closure in these experiments is as a disambiguating image property, a factor which complements the influence of proximity in determining the perceptual organization of the image.

To test this idea, we repeated the original experiments with closed and open stimuli, but with a $50 \%$ increase in inter-stimulus spacing. The results (Fig. 18) show that substantially decreasing the proximity of neighboring stimuli has no significant effect on search speed. (For the closed stimuli, slope $=16 \mathrm{msec} / \mathrm{item}$, intercept $=548 \mathrm{msec} /$ item. For the open stimuli, slope $=95 \mathrm{msec} / \mathrm{item}$, intercept $=240 \mathrm{msec} / \mathrm{item}$.) ${ }^{*}$

While interpretation of this result is complicated by the simultaneous increase in total display size (from $7 \times 7^{\circ}$ to $10.5 \times 10.5^{\circ}$ ), it does suggest that closure has a larger role to play in perceptual organization than as a simple counteracting factor to proximity. In other words, closure seems to determine the speed with which the two-dimensional shape representation of a single outline figure can be formed.

\section{CONCLUSION}

The smooth relationship between closure and shape can be seen to balance two demands. While it is critical that perceptual processes be robust to contour fragmentation caused by occlusion or weak edge contrast, it is equally important that contour fragments arising from separate object boundaries or surface markings not be

*Although there was no significant difference in search slopes $(P>0.1)$, increasing the inter-stimulus spacing did significantly decrease the intercept for the open stimuli $(P<0.05)$.

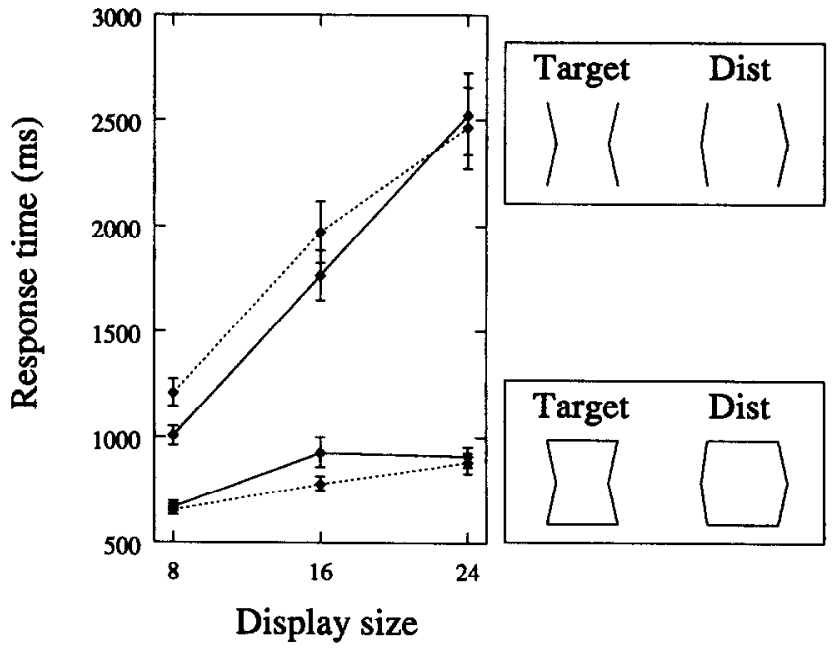

FIGURE 18. Search results for sparse stimuli (14 subjects). Results for closed and open stimuli at the standard density are shown dotted for reference.

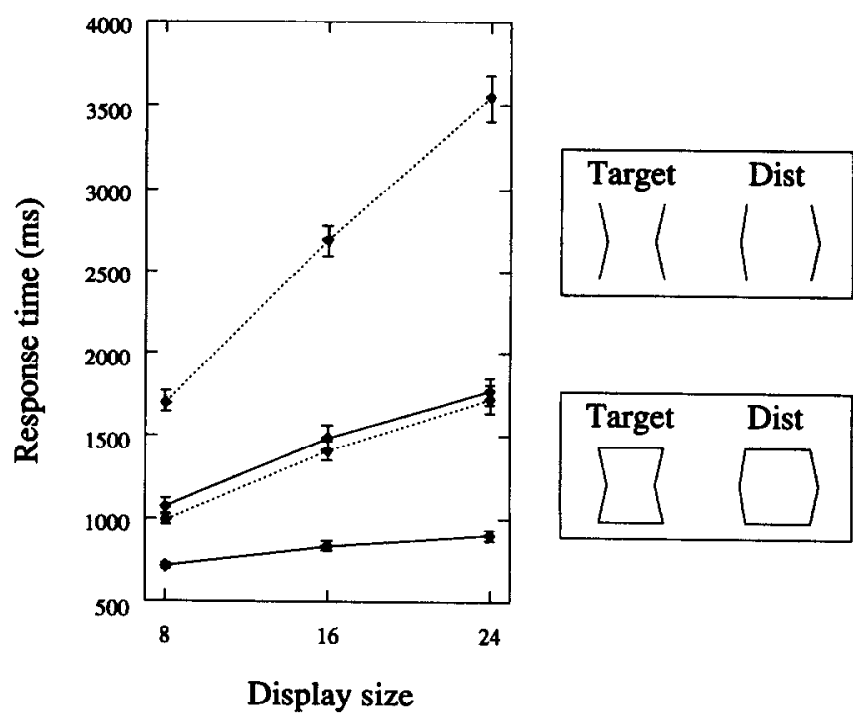

FIGURE 19. Visual search results using the classical procedure (14 subjects). Target-present results are shown solid, target-absent results are shown dotted. 
incorrectly grouped as projections of a single object boundary. The topological concept of a closed curve is too rigid to meet these demands. Rather, the notion of a closure continuum seems to be key in the perceptual processing of two-dimensional shape.

\section{REFERENCES}

Biederman, I. (1988). Aspects and extensions of a theory of human image understanding. In Pylyshyn, Z. (Ed.), Computational processes in human vision (pp. 370-427). Norwood, N.J.: Ablex.

Blum, H. (1973). Biological shape and visual science (Part I). Journal of Theoretical Biology, 38, 205-287.

Caelli, T. M., Julesz, B. \& Gilbert, E. N. (1978). On perceptual analyzers underlying visual texture discrimination: Part II. Biological Cybernetics, 29, 201-214.

Cave, K. R. \& Wolfe, J. M. (1990). Modeling the role of parallel processing in visual search. Cognitive Psychology, 22, 225-271.

Chun, M. M., Wolfe, J. M. \& Friedman-Hill, S. R. (1991). Just say no: How to terminate a visual search. Investigative Ophthalmology and Visual Science, 32, 1041.

Donnelly, N., Humphreys, G. W. \& Riddoch, M. J. (1991). The parallel computation of primitive shape descriptions. Journal of Experimental Psychology: Human Performance and Perception, 17, 561-570.

Elder, J. (1992). Contour closure and the perception of shape. Master's thesis, Department of Electrical Engineering, McGill University, Canada.

Enns, J. T. \& Rensink, R. A. (1991). Preattentive recovery of threedimensional orientation from line drawings. Psychological Review, 98, 335-351.

Glass, L. \& Switkes, E. (1976). Pattern recognition in humans: Correlations which cannot be perceived. Perception, 5, 67-72.

Grossberg, S. \& Mingolla, E. (1985). Neural dynamics of form perception: Boundary completion, illusory figures, and neon color spreading. Psychological Review, 92, 173-211.

Hoffman, D. D. \& Richards, W. A. (1985). Parts of recognition. Cognition, 18, 65-96.

Julesz, B. (1980). Spatial nonlinearities in the instantaneous perception of textures with identical power spectra. Philosophical Transactions of the Royal Society of London B, 290, 83-94.

Julesz, B. (1986). Texton gradients: The texton theory revisited. Biological Cybernetics, 54, 245-251.

Julesz, B. \& Bergen J. R. (1987). Textons: The fundamental elements in preattentive vision and perception of textures. In Fischler, M. A. \& Firschein, O. (Eds), Readings in computer vision (pp. 242-256). Altos, Calif.: Kaufmann.

Kimia, B., Tannenbaum, A. \& Zucker, S. W. (1990). Toward a computational theory of shape: An overview. Proceedings of the First European Conference on Computer Vision, 427, 402-407.

Koenderink, J. J. (1984). What does the occluding contour tell us about solid shape? Perception, 13, 321-330.

Koffka, K. (1935). Principles of Gestalt psychology. New York: Harcourt, Brace \& World.

Leyton, M. (1989). Inferring causal history from shape. Cognitive Science, 13, 357-387.

Lowe, D. G. (1985). Perceptual organization and visual recognition. Boston, Mass.: Kluwer.

Marr, D. (1982). Vision. New York: (W. H.) Freeman.

Pomerantz, J. R., Sager, L. C. \& Stoever, R. G. (1977). Perception of wholes and their component parts: Some configural superiority effects. Journal of Experimental Psychology: Human Performance and Perception, 3, 422-435.

Prazdny, K. (1983). Illusory contours are not caused by simultaneous brightness contrast. Perception and Psychophysics, 34, 403-404.

Prazdny, K. (1986). Psychophysical and computational studies of random-dot Moire patterns. Spatial Vision, 1, 231-242.

Rock, I. \& Palmer, S. (1990). The legacy of Gestalt psychology. Scientific American, 263, 84-91.
Shapley, R. \& Gordon, J. (1985). Nonlinearity in the perception of form. Perception and Psychophysics, 37, 84-88.

Treisman, A. \& Gelade, G. (1980). A feature integration theory of attention. Cognitive Psychology, 12, 97-136.

Treisman, A. \& Gormican, S. (1988). Feature analysis in early vision: Evidence from search asymmetries. Psychological Review, 95, 15-48.

Treisman, A. \& Paterson, R. (1984). Emergent features, attention and object perception. Journal of Experimental Psychology: Human Perception and Performance, 10, 12-31.

Zucker, S. W. (1986). The diversity of perceptual grouping. In Arbib M. \& Hanson, A. (Eds), Vision, brain and cooperative computation (pp. 231-261). Cambridge, Mass.: MIT Press.

Zucker, S. W. \& Davis, S. (1988). Points and endpoints: A size/spacing constraint for dot grouping. Perception, 17, 229-247.

Zucker, S. W., Stevens, K. A. \& Sander, P. (1983). The relation between proximity and brightness similarity in dot patterns. Perception and Psychophysics, 34, 513-522.

Acknowledgements -We thank Allan Dobbins, Greg Dudek, Thomas Jelonek, David Jones, Benjamin Kimia, Michael Langer, Michael Morgan, Ron Rensink, Frances Wilkinson, and two anonymous reviewers for useful comments on this work.

\section{APPENDIX}

In the traditional visual search technique (Treisman \& Gelade, 1980), subjects are shown an equal mix of displays with a target and displays without a target. Subjects press one button when they have detected a target in the display, another if they are unable to find a target. But if a subject is having problems finding a target, how does he or she decide when to stop looking? One suggestion is that subjects use a rough timing mechanism based upon how difficult they expect the task to be (Chun, Wolfe \& Friedmun-Hill, 1991).

Although subjects rarely indicate that a target is present when it is not (target absent error rates are seldom more than 10\% and typically average about $5 \%$ ), it is much more common for subjects to indicate that the target is absent when in fact it is present in the display (target present error rates often average more than $10 \%$ and can be as high as $20 \%$ or even $30 \%$ ) (Donnelly, Humphreys \& Riddoch, 1991; Enns \& Rensink, 1991). Moreover, this error rate has been observed to correlate positively with reaction time (Enns \& Rensink, 1991). Thus, to use the timer analogy, when the task is difficult, subjects time-out more often.

Of course when an error is made, the trial is not used in determining the mean reaction time for the associated stimulus and display size. This means that these estimates will be systematically depressed. Worse yet, this depression will be greater for conditions which are harder: larger display sizes with less easily discriminable stimuli. This results in a general depression of search slope estimates, particularly for more difficult tasks.

The size of the variance under particular stimulus conditions is normally quite large in visual search experiments. For example, second-order fits to the response-time data of our experiments indicate that less than one third of the variance for a particular stimulus type is due to variation in display size. Moreover, this

TABLE 2. Linear fit parameters for search results using the classical procedure

\begin{tabular}{llccc}
\hline Procedure & Closure & Target status & $\begin{array}{c}\text { Slope } \\
\text { (msec/item) }\end{array}$ & $\begin{array}{c}\text { Intercept } \\
\text { (msec) }\end{array}$ \\
\hline Classical & Closed & Present & 12 & 621 \\
Classical & Closed & Absent & 48 & 609 \\
Classical & Open & Present & 45 & 727 \\
Classical & Open & Absent & 116 & 786 \\
Unbiased & Closed & Present & 14 & 546 \\
Unbiased & Open & Present & 83 & 555 \\
\hline
\end{tabular}




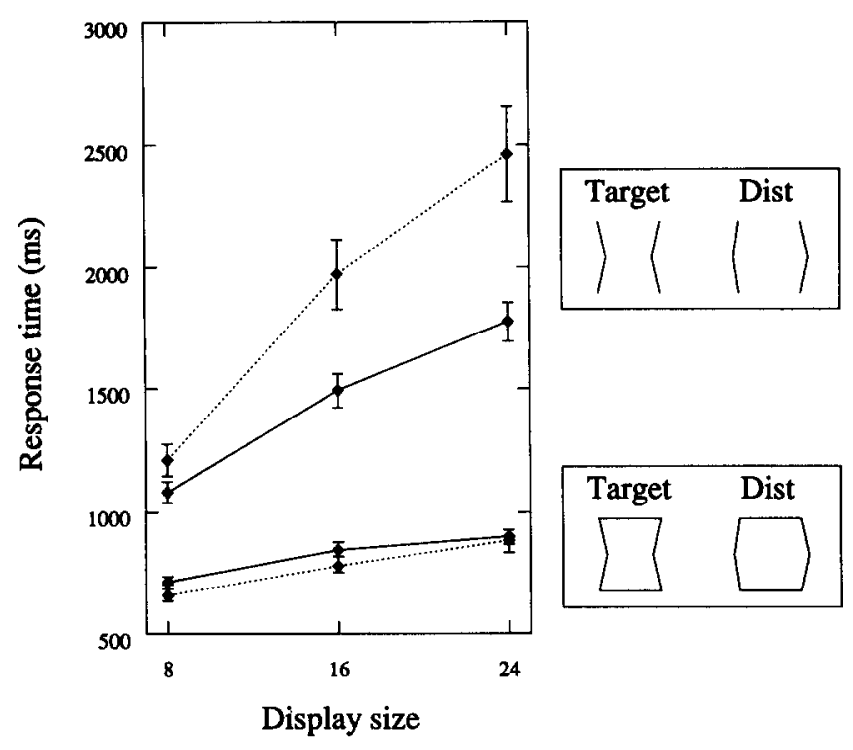

FIGURE 20. Comparison of visual search results using the different experimental procedures. Results using the classical procedure are shown solid. Results using our procedure are shown dotted.

unmodeled variance increases with response time. This means that the biases caused by the disqualification of hard trials could be very significant.

The visual search technique used in this work overcomes the limitations of the classical procedure. This is accomplished primarily by eliminating the uncertainty associated with classical visual search: in our technique the subjects know that the target will always be somewhere in the display. What they do not know is where. Thus subjects keep looking until they find the target: they never time-out.

By removing this dominant source of error in target present displays, we have achieved error rates averaging $<2 \%$. Thus even if there remains some small correlation between error rate and response time, the error rate is too low for the resulting bias to be significant.

*Search slope for the closed stimuli is significantly less than that for the open stimuli $(P<0.005)$. Intercepts do not differ significantly $(P>0.1)$.

†For the closed figures, neither slope nor intercept differ significantly between methods $(P>0.1)$. For the open figures, both slope $(P<0.005)$ and intercept $(P<0.05)$ are significantly lower for the classical procedure than for our procedure.

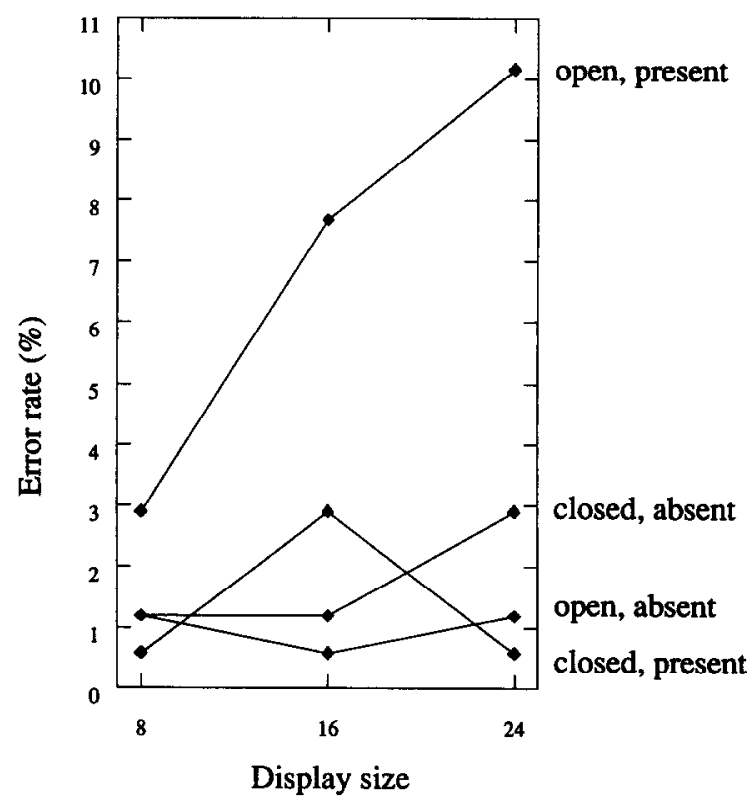

FIGURE 21. Mean error rates for the classical visual search procedure.

In order to both validate the technique and test for bias, we repeated the basic closure experiment of Section 4 using the classical procedure The results (Fig. 19) confirm that search is rapid for closed stimuli but slow for open stimuli.*

Table 2 shows the linear model parameters for the results using the classical procedure and the results using our procedure (Section 4). Figure 20 shows the target-present results for the classical procedure plotted with the results using our procedure. While the results for the closed stimuli do not differ significantly, search for the open figures appears to be faster using the classical procedure. $\dagger$

An examination of the error rates for these experiments may shed light on this difference. The error rates using our technique remained under $3 \%$, averaging $1 \%$ for the closed stimuli and $1.8 \%$ for the open stimuli. The error rates using the classical technique are shown in Fig. 21. Again, error is below 3\% except for the target-present condition of the difficult trials: display sizes of 16 and 24 with open stimuli resulted in error rates of 7.7 and $10.2 \%$ respectively.

Thus, as predicted, the classical technique leads to target-present error rates which are strongly correlated with response time and which become significant for the large display sizes of hard tasks. We believe that this accounts for the depressed search slope observed for the open stimuli using the classical visual search procedure (Fig. 20). 